\title{
Oral Health Case Reports
}

\section{Swelling Left Medial Canthus}

\section{Schepman KP* and Eshghi O}

University Medical Center Groningen, Netherlands

*Corresponding author: Schepman KP, Department of Oral diseases, Jaw and Facial Surgery at the University Medical Center Groningen, Hanzeplein 1, 9713 GZ Groningen, Netherlands, Tel: +31503616161; E-mail: k.p.schepman@umcg.nl

Rec date: Mar 01 2016; Acc date: Apr 09, 2016; Pub date: Apr 14, 2016

Copyright: (C) 2016 Schepman KP, et al. This is an open-access article distributed under the terms of the Creative Commons Attribution License, which permits unrestricted use, distribution, and reproduction in any medium, provided the original author and source are credited.

\section{Introduction}

A 73 year old man was referred to our hospital because of intermitting hemorrhage from a swelling of the left medial orbital canthus (Figure 1).

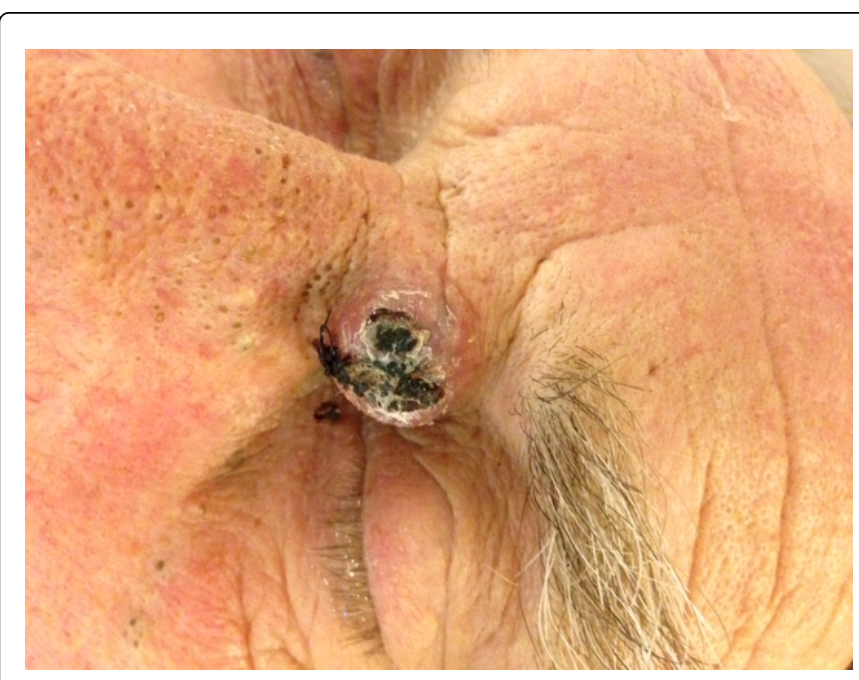

Figure 1: Swelling of the left medial orbital canthus.

Patient told he suffered from a small soft elastic, canthal swelling for several months. On the assumption of the clinical diagnosis "lipoma" an excision was performed. However, a large swelling developed and several bleedings occurred out of the lesion. Imaging (CT) showed a vascular malformation (Figure 2).

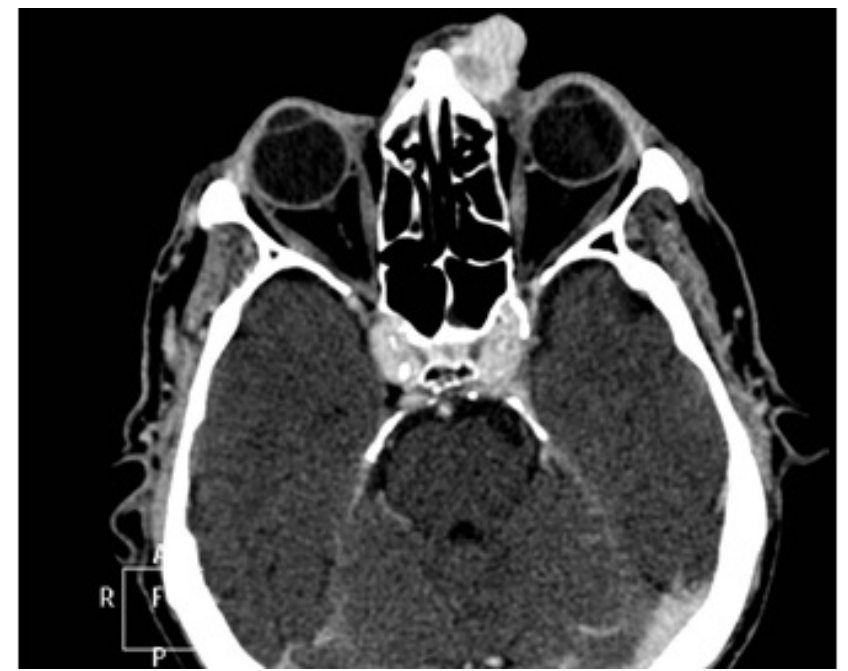

Figure 2: Vascular malformation.

Patient was transferred to our hospital for embolization. Angiography showed a large arterial venous fistula with venous pouch primarily fed by the ophthalmic artery (from both sides), and to a lesser extent by the ethmoidal and maxillar artery (Figure 3).

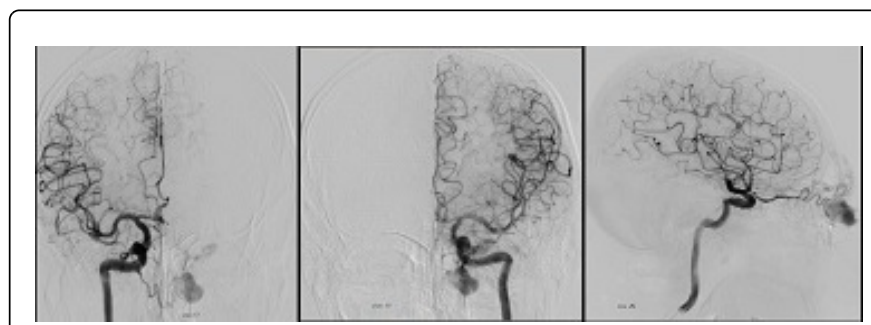

Figure 3: Ethmoidal and maxillar artery.

Venous drainage of the pouch ran through the superior orbital vene to the cavernous sinus on both sides. Embolization of the venous pouch was performed by injecting glue directly into the venous pouch until the whole pouch was filled (Figure 4). 
Page 2 of 2

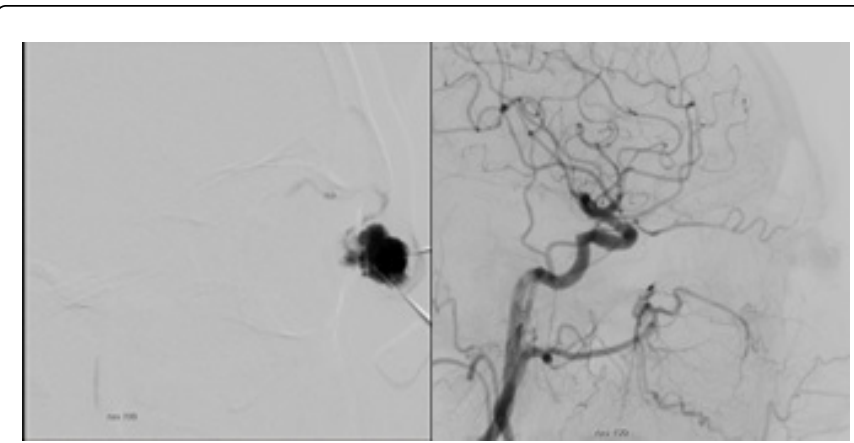

Figure 4: Embolization of the venous pouch.
Therefore two 19 gauge winged infusion needles (Terumo Europe, Leuven Belgium) were positioned into the venous pouch. One of the needles were used for injection of histhoacryl which was diluted with lipiodol (Guerbert, Aulnay-Sous-Bois, France) with a concentration of 1 to one and the second needle were used for injection of histoacryl one to 3 diluted with lipiodol. The strongest dilution (one to 1 ) was used to embolize the reflux to cavernous sinus to prevent cavernous sinus embolization. The second needle were used to embolize the whole collateral venous network around the venous pouch.

The complete embolization of the fistula were confirmed by injection of both common carotid arteries. The lesion in the left orbital canthus was completely in regression one month after intervention. The history of instant progression of the swelling after operation, as well as the normal caliber of the arterial inflow suggested a probably iatrogenic arterial venous fistula. 